\title{
Prefrontal cortex and the organization of recent and remote memories: An alternative view
}

\author{
Jerry W. Rudy, ${ }^{1}$ Joseph C. Biedenkapp, and Randall C. O'Reilly \\ Center for Neuroscience and Department of Psychology, University of Colorado, Boulder, Colorado 80309, USA
}

Since Marr's (1971) classic work, theories of the hippocampus have been guided by four assumptions.

1. The hippocampus rapidly and automatically acquires information.

2. The hippocampus is a limited capacity storage system and consequently is subject to relatively rapid forgetting.

3. Information originally stored in the hippocampus becomes permanently stored in the cortex and independent of the hippocampus.

4. The hippocampus plays a critical role in the genesis of the cortical memories.

The last three assumptions form the basis of what is called the standard theory of systems consolidation (Squire and Alvarez 1995). The major premise of this theory is that there is a dynamic reorganization in the location of memories as a function of their age. As new memories are formed, old memories get moved from the hippocampus to cortex. In its strong form, this theory predicts that, provided enough time, memories once dependent on the hippocampus can survive even if the hippocampus is completely removed. Numerous studies of humans and other animals with damage to the hippocampus have been conducted to evaluate this view.

Recently, Bontempi, Frankland, and their colleagues (Bontempi et al. 1999; Frankland et al. 2004; Maviel et al. 2004) have applied a different methodology to investigate the retention of new and old memories. They measured neuronal activity in different brain regions induced by a retention test of memories thought to initially depend on the hippocampus. Their important finding was that activity in the hippocampus and regions of the prefrontal cortex (PFC) changed significantly as a function of the age of the memory. For recently acquired memories, there was high activity in the hippocampus and low activity in the PFC regions, whereas the opposite pattern was observed for older memories (Fig. 1).

In their recent review work, Frankland and Bontempi (2005) interpreted these results in the context of the standard theory of systems consolidation, suggesting that PFC regions play the same role in retrieving old memories that hippocampus plays in retrieving new memories. Thus, memories initially acquired in the hippocampus become consolidated into the PFC and other cortical areas, with the PFC being a critical locus of consolidation because of its broad patterns of interconnectivity with other brain areas (which is similar to that of the hippocampus). Their hypothesis accounts for why the patterns of regional brain activity depend on the age of the memory and why inactivating the relevant PFC regions has no effect on retrieving a new memory but impairs retrieval of an old memory (Frankland et al. 2004; Maviel et al. 2004).

\footnotetext{
'Corresponding author.

E-mail: JRudy@Clipr.Colorado.Edu; fax: (303) 492-2967.

Article published online ahead of print. Article and publication date are at http://www.learnmem.org/cgi/doi/10.1101//m.97905.
}

\section{Alternative theory}

We suggest an alternative explanation that leverages existing theories that emphasize the importance of PFC for cognitive control and top-down attention (O'Reilly et al. 1999; Miller and Cohen 2001). Specifically, we argue that the differential involvement of the PFC in older memories simply reflects the consequences of natural forgetting. Due to either decay or interference, older memories become weaker and more difficult to retrieve. These weaker memories require additional activation from the PFC to be retrieved.

There is considerable evidence from the human literature that PFC plays an important role in strategic activation and recall of memories (Fletcher and Henson 2001; Miyashita 2004; Ranganath et al. 2004). Furthermore, mechanisms have been proposed that enable the PFC to be activated in response to a potential retrieval failure. For example, weak (old) memories may lead to diffuse patterns of neural activation that trigger conflict detectors in the anterior cingulate cortex (Botvinick et al. 2001). Indeed, Frankland and Bontempi's work (Bontempi et al. 1999; Frankland et al. 2004; Maviel et al. 2004) revealed that this region becomes active when mice were retrieving old memories.

According to our view, PFC activity does not represent the activation of a cortical component of the old memory trace that has been consolidated into this region. Instead, it represents activation in response to a potential failure of the retrieval cues to access the degraded memory trace. In this case, the PFC can provide a signal to boost activity in regions that contain the trace. New memories are unaffected when PFC is inactivated because they have not degraded and are easily retrieved through hippocampal memory traces (see Fig. 2).

In support of the idea that hippocampal memory traces degrade with age, Frankland, Bontempi and colleagues (Bontempi et al. 1999; Frankland et al. 2004; Maviel et al. 2004) reported that activation in the hippocampus was significantly less when the retrieved memory was old than when it was new (see Fig. 1). Although forgetting was not always revealed by the behavioral measures used in their studies, there was clear forgetting in the Bontempi et al. (1999) experiment. Where forgetting was absent, we would argue that the activity boost provided by PFC enabled successful retrieval. Furthermore, we suggest that more subtle measures of forgetting would reveal that the old memory trace had degraded. For example, it is known that as a memory ages, it can be retrieved by a broader range of stimulus cues (i.e., the generalization gradient broadens; Riccio et al. 1992).

These two accounts of the contribution of the PFC can be empirically evaluated. For example, a key implication of our account is that the age of the memory is a correlate, but not the critical variable determining when the PFC will be activated. Instead, it is the strength of the memory trace. To the degree the trace is degraded or weak, PFC neurons will be activated. Thus, it should be possible to reverse the age-related changes in PFC activation and the effects of inactivating PFC at the time of retrieval. One can evaluate this idea by testing retrieval of a weak new memory and a strong old memory. Our account predicts 


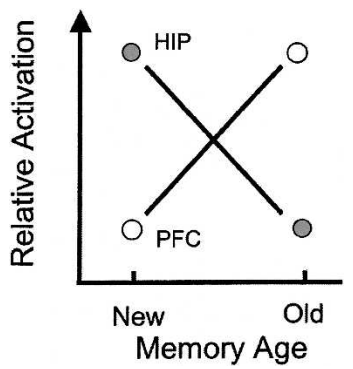

Figure 1. The relative neuronal activity in the hippocampus and prefrontal cortex during retention as a function of the age of the memory (based on the collective results of Bontempi et al. 1999; Frankland et al. 2004; Maviel et al. 2004). In the Frankland and Bontempi (2005) model, activity in the hippocampus and PFC are inversely related, because as the memory ages, the memory trace moves into the PFC (see Frankland and Bontempi 2005).

that PFC will be active when the new memory is weak in comparison to when the memory is old but strong.

Consistent with our position, Bunge et al. (2004) have recently reported fMRI data with people that support our contention that the strength of the memory may be an important dimension regulating ACC. They directly manipulated memory strength of visual associations by varying the number of encoding trials. They found that activation in ACC and hippocampus was negatively correlated on trials with weak associations. When activity in hippocampus was low, activity in ACC was high. This pattern suggests that ACC was recruited when the recollection processes dependent on the hippocampus needed a boost. Based on their analysis of activation patterns associated with weak and strong visual associations, and other data, Bunge et al. (2004) suggested that, "These convergent findings suggest that this subregion within ACC is differentially engaged under retrieval conditions in which recollection is poor". It is interesting to note that Thomas et al. (2002) also have suggested that the engagement of anterior cingulate during retrieval of fear memories may be related to the strength of the fear memory.

Our account also raises the important question of how the PFC is informed that there is a potential retrieval failure. What is the signal that activates PFC when memory is about to fail and how does it promote successful retrieval? We have suggested that it may be an ACC-mediated conflict-like signal, but there may be other alternatives that could be explored.

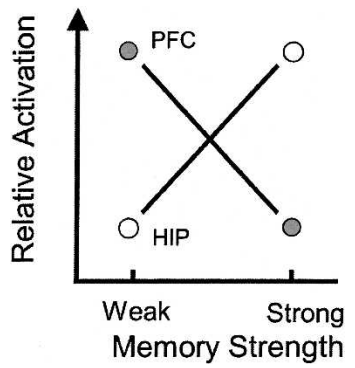

Figure 2. The theoretical relationship between neuronal activity in the hippocampus and PFC during retention. According to our view, activation in the hippocampus and PFC are inversely related because neurons in PFC become activated when there is a potential for a retrieval failure. This potential would be determined by the strength of the memory trace and can be independent of the age of a memory. Weak traces require a boost from the PFC in comparison to strong traces.

\section{Conclusion}

Frankland and Bontempi (2005) have offered an interesting and new conception of the role of PFC regions in the retention and retrieval of new and old memories. We hope that by offering an alternative account that is consistent with considerable existing data about the function of the PFC, that researchers will be motivated to experimentally evaluate the different implications of the two views. If the Frankland and Bontempi (2005) view is correct, then the standard model of systems consolidation would be greatly strengthened. If we are correct, however, the standard theory of systems consolidation draws no support from the Frankland and Bontempi studies (Bontempi et al. 1999; Frankland et al. 2004; Maviel et al. 2004).

We have offered an alternative to Frankland and Bontempi's systems consolidation hypothesis that assumes that the content of the memory is transferred to the PFC as the memory ages. However, although we question their account, it should be appreciated that our hypothesis-that PFC facilitates the retrieval of weak memories in other regions of the brain-is in no way incompatible with the general idea that as memories age they can become independent of the hippocampus.

\section{References}

Bontempi, B., Laurent-Demir, C., Destrade, C., and Jaffard, R. 1999. Time dependent reoganization of brain circuitry underlying long-term memory storage. Nature 400: 671-674.

Botvinick, M.M., Braver, T.S., Barch, D.M., Carter, C.S., and Cohen, J.D. 2001. Conflict monitoring and cognitive control. Psychol. Rev. 108: $624-652$.

Bunge, S.A., Burrows, B., and Wagner A.D. 2004. Prefrontal and hippocampal contributions to visual associative recognition: Interactions between cognitive control and episodic retrieval. Brain Cogn. 56: 141-152.

Fletcher, P.C. and Henson, R.N.A. 2001. Frontal lobes and human memory. Insights from functional neuroimaging. Brain 124: $849-881$.

Frankland, P.W. and Bontempi, B. 2005. The organization of recent and remote memories. Nat. Rev. Neurosci. 6: 119-130.

Frankland, P.W., Bontempi, B., Talton, L.E., Kaczmarek, L., and Silva, A.J. 2004. The involvement of the anterior cingulate cortex in remote contextual fear memory. Science 304: 881-883.

Marr, D. 1971. Simple memory: A theory for archicortex. Philos. Transr. Soc. Lond. B. 262: 23-81.

Maviel, T., Durkin, T.P., Menzaghi, F., and Bontempi, B. 2004. Sites of neocortical reorganization critical for remote memory. Science 305: 96-99.

Miller, E.K. and Cohen, J.D. 2001. An integrative theory of prefrontal cortex function. Annu. Rev. Neurosci. 24: 167-202.

Miyashita, Y. 2004. Cognitive memory: Cellular and network machineries and their top-down control. Science 306: 435-440.

O'Reilly, R.C., Braver, T.S., and Cohen, J.D. 1999. A biologically based computational model of working memory. In Models of working memory: Mechanisms of active maintenance and executive control. (eds. A. Miyake and P. Shah), pp. 375-411. Cambridge University Press, New York.

Ranganath, C., Cohen, M.X., Dam, C., and Desposito, M. 2004. Inferior temporal, prefrontal, and hippocampal contributions to visual working memory maintenance and associative memory retrieval. $J$. Neurosci. 24: 3917-3943.

Riccio, D.C., Ackil, J.K., and Burch-Vernon, A. 1992. Forgetting of stimulus attributes: Methodological implications for assessing associative phenomena. Psychol. Bull. 112: 433-445.

Squire, L.R. and Alvarez, P. 1995. Retrograde amnesia and memory consolidation: A neurobiological perspective. Curr. Opin. Neurobiol. 5: $169-177$.

Thomas, K.L., Hall, J., and Everitt, B.J. 2002. Cellular imaging with zif268 expression in the rat nucleus accumbens and frontal cortex further dissociates the neural pathways activated following the retrieval of contextual and cued fear memory. Eur. J. Neurosci. 16: $1789-1796$. 


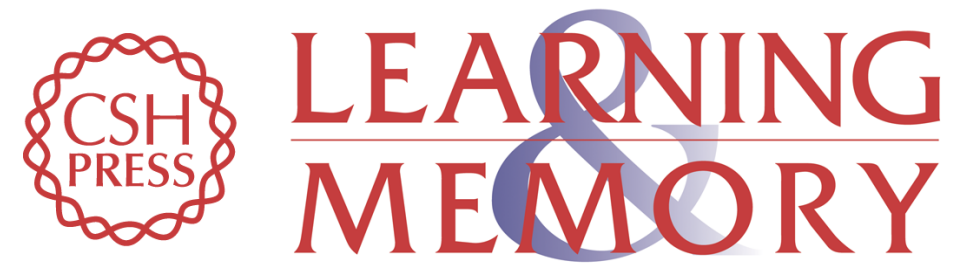

\section{Prefrontal cortex and the organization of recent and remote memories: An alternative view}

Jerry W. Rudy, Joseph C. Biedenkapp and Randall C. O'Reilly

Learn. Mem. 2005, 12:

Access the most recent version at doi:10.1101/lm.97905

References This article cites 14 articles, 4 of which can be accessed free at:

http://learnmem.cshlp.org/content/12/5/445.full.html\#ref-list-1

License

Email Alerting Receive free email alerts when new articles cite this article - sign up in the box at the Service top right corner of the article or click here. 